\title{
Anisotropic pinning and the mixed-state galvanothermomagnetic properties of superconductors - a phenomenological approach
}

\author{
V. A. Shklovskij \\ National Science Center - «Kharkov Institute of Physics and Technology», Institute of Theoretical Physics. \\ 1 Akademicheskaya St., 310108, Kharkov, Ukraine \\ Kharkov State University, Physical Department, 4 Svobody Sq., 310077, Kharkov, Ukraine
}

Submitted July 4, 1997, revised July 10, 1997

\begin{abstract}
In the presence of isotropic and anisotropic pinning the vortex dynamics is discussed in terms of phenomenologically introduced, nonlinear viscosities. The formulas for linear galvanothermomagnetic effects are derived and analyzed under the condition at which the transport current or temperature gradient is directed at arbitrary angle with respect to the unidirected twins, which cause the anisotropic pinning. It is shown that two new effects which appear due to the anisotropic pinning, namely (with respect to the reversal of the magnetic field direction) even transverse and odd longitudinal voltage, have a distinct origin. The first is due to the guided vortex motion, while the second appears only when anisotropic (in contrast with isotropic) pinning changes the Hall drag coefficient. We also show that the last effect might be masked in the experiment by a large, odd contribution, which has the same angular dependence and which appears due to the Ettingshausen effect. In order to clarify the problem of influence of the twins on the Hall drag coefficient, we discuss the possibility of separating these two contributions in the experiment.
\end{abstract}

PACS: 74.25.Fy, 74.60.Ge

1. The influence of the pinning on the transport properties of high- $T_{c}$ superconductors (HTSC) is a very interesting problem. One of the open and rather controversial issues in this field is the influence of the pinning on the Hall drag coefficient. Recently, Vinokur et al. [1] have calculated the effect of point (isotropic) pins on the Hall resistivity and showed that the Hall constant is pinning-independent. Is it also true for the anisotropic pinning caused, for example, by a system of unidirected twins in YBCO single crystal? Sonin et al. [2] have shown that in the last case, in addition to the usual longitudinal and transverse (Hall) resistivities (even and odd, accordingly, with respect to the reversal of a magnetic field direction) two new contributions to the resistivity appear: the even transverse and the odd longitudinal contributions. These results follow in Ref. 2 from the general form of the linear Ohm's law in anisotropic media which, in turn, was formulated on the basis of symmetry considerations. In fact, it was postulated in Ref. 2 that presence of the twins changes the Hall conductivity. The experimental situation also is still con- troversial. Recent paper [3] claims that for a twinned (and further irradiated with high-energy $\mathrm{Pb}$ ions) YBCO single crystal the mixed-state Hall conductivity does not depend on the pinning strength, in complete agreement with the theory [1].

The main purpose of this paper is to suggest and develop theoretically a new method for experimental verification of the plausible effect of twins on the Hall drag coefficient within the framework of the phenomenological approach used earlier in Ref. 1 for the case of isotropic pinning. We modify the method of Ref. 1 for considering both the isotropic and anisotropic pinning so that we can derive the Ohm's law, which was postulated in Ref. 2. We can therefore clarify the origin of the earlier introduced [2] four phenomenological resistivities in terms of the drag and pinning vortex viscosities, i.e., on a more detailed level. In the linear case we show that the above-mentioned, new, even, transverse contribution is due completely to the guided vortex motion [4] and does not require the modification of the Hall drag coefficient by twins, whereas the odd longitudinal contribution depends entirely on the 
different values of this coefficient for motion of vortices along the twins and across them. It follows from this conclusion that in order to justify the influence of the twins on the Hall drag coefficient, we must identify only odd longitudinal contribution in the measurements.

But as we show below, experimental observation of this small (Hall in nature) contribution may be masked by the possible appearance (due to the emergence of a small temperature gradient in the presence of the transport current) of the odd Seebeck contribution with the same angular dependence. In order to give a theoretical basis for the separation of these two contributions, we also calculated the thermomagnetic properties of the sample with anisotropic pinning, using the same approach. We will show that the main contribution to the odd Seebeck resistivity now gives the guided vortex motion, while the possible Hall contribution is small and can be disregarded.

2. Following Ref. 1, we have for the average velocity of vortices $\mathbf{v}$ the equation of motion

$$
\eta \mathbf{v}+\alpha \mathbf{v} \times \mathbf{n}=\mathbf{f}+\mathbf{f}_{p},
$$

where $\eta$ is the isotropic friction coefficient, $\alpha$ is the isotropic Hall drag viscosity, $\mathbf{f}$ is the moving external force (Lorentz or thermal, see below), and $\mathbf{f}_{p}$ is the average resulting pinning force, which is the sum of the isotropic contribution $\mathbf{f}_{p}^{i}$ and the anisotropic contribution $\varepsilon \mathbf{f}_{p}^{a}$, where $\varepsilon=a_{0} / d$ is relative fraction of vortices placed on the twins ( $a_{0}$ and $d$ are the average distances between vortices and twins, respectively). Considerations which may lead to the equation of motion in the form (1) will be discussed in detail elsewhere. If $\varepsilon=0$, we obtain Eq. (2) in Ref. 1. We assume that the average anisotropic pinning force $\mathbf{f}_{p}^{a}$ can be decomposed into two parts $\mathbf{f}_{p}^{a}=\mathbf{f}_{p}^{t}+\mathbf{f}_{p}^{l}$, where, neglecting small Hall viscosities, $\mathbf{f}_{p}^{t}=f_{p}^{t} \cdot \mathbf{m}, \mathbf{f}_{p}^{l} \equiv f_{p}^{l} \cdot \mathbf{m}_{\|}$. Here $\mathbf{m}$ and $\mathbf{m}_{\|} \equiv \mathbf{z} \times \mathbf{m}$ are the unit vectors directed perpendicularly and parallel to the twins, respectively, $\mathbf{z}$ is the unit vector which is perpendicular to the sample's plane, $f_{p}^{t} \equiv \mathbf{f}_{p}^{t} \cdot \mathbf{m}$, and $f_{p}^{l} \equiv \mathbf{f}_{p}^{l} \cdot \mathbf{m}_{\|}$. As in Ref. 1 , we assume that $\mathbf{f}_{p}^{i}=-\gamma_{i}(\tau) \mathbf{v}$, where $\gamma_{i}(\tau)>0$ is phenomenological coefficient, which depends only on the value of $v \equiv|\mathbf{v}|$. For $\mathbf{f}_{p}^{t}$ and $\mathbf{f}_{p}^{l}$ we assume that

$$
\begin{aligned}
& \mathbf{f}_{p}^{t}=-\gamma_{t}\left(\left|v_{t}\right|\right) \mathbf{v}_{t}-\alpha_{t} \mathbf{v}_{t} \times \mathbf{n}, \\
& \mathbf{f}_{p}^{l}=-\gamma_{l}\left(|v|_{l}\right) \mathbf{v}_{l}-\alpha_{l} \mathbf{v}_{l} \times \mathbf{n},
\end{aligned}
$$

where $\gamma_{t}$ and $\gamma_{l}$ are the average phenomenological anisotropic viscosities which include also the $\mathbf{v}$-independent terms for the motion of vortices across the twins and along them, respectively; $\alpha_{t}$ and $\alpha_{l}$ are the corresponding anisotropic Hall drag coefficients, and $\mathbf{n}$ is the unit vector in the magnetic field direction $(\mathbf{n}=n \mathbf{z}$, where $n= \pm 1)$. Here $\mathbf{v}_{l}=v_{l} \mathbf{m}_{\|}$ and $\mathbf{v}_{t}=v_{t} \mathbf{m}$ are longitudinal and transverse vortex velocities, respectively $\left(\mathbf{v}=\mathbf{v}_{l}+\mathbf{v}_{t}\right)$. Below we will show that incorporation of the Hall terms in (2) is equivalent to the assumption that the anisotropic pinning influences the Hall drag coefficient $\alpha$ and leads to a new effect - the odd longitudinal contribution to the resistivity of the sample.

Substitution of the expressions for $\mathbf{f}_{p}$ into (1) leads to a system of two nonlinear equations for $v_{t}$ and $v_{l}$

$$
\left\{\begin{aligned}
\eta_{t} v_{t}+n \alpha_{H l} v_{l} & =f_{t}, \\
-n \alpha_{H t} v_{t}+\eta_{l} v_{l} & =f_{l},
\end{aligned}\right.
$$

where

$$
\begin{gathered}
\eta_{t} \equiv \eta+\gamma_{i}(v)+\varepsilon \gamma_{t}(|v|), \\
\eta_{l} \equiv \eta+\gamma_{i}(v)+\varepsilon \gamma_{l}\left(\left|v_{l}\right|\right), \\
\alpha_{H l} \equiv \alpha+\varepsilon \alpha_{l}, \alpha_{H t} \equiv \alpha+\varepsilon \alpha_{t},
\end{gathered}
$$

and $f_{t} \equiv \mathbf{f} \cdot \mathbf{m}$ and $f_{l} \equiv \mathbf{f} \cdot \mathbf{m}_{\|}$are the transverse and longitudinal components of the external force, respectively.

In the linear case, where $\eta_{t}$ and $\eta_{l}$ are constants, i.e., they do not depend on the velocities, the solution of (3) is simple

$$
\begin{gathered}
v_{t}=\Delta^{-1}\left(\eta_{l} f_{t}-n \alpha_{H} f_{l}\right) ; \quad v_{l}=\Delta^{-1}\left(\eta_{t} f_{l}+n \alpha_{H t} f_{t}\right) ; \\
\Delta \equiv \eta_{t} \eta_{l}+\alpha_{H l} \alpha_{H t} .
\end{gathered}
$$

The electric field induced by the vortex motion is $\mathbf{E}=(1 / c) \mathbf{B} \times \mathbf{v}$, and if the external force is equal to the Lorentz force $\mathbf{f}=\left(\Phi_{0} / c\right) \mathbf{j} \times \mathbf{n}$, where $\Phi_{0}$ is the flux quantum, $c$ is the light velocity, and $\mathbf{j}$ is the transport current density, then from (5) we obtain

$$
\begin{gathered}
\mathbf{E}_{L}=\rho_{t} \mathbf{m}_{\|}\left(\mathbf{m}_{\|} \cdot \mathbf{j}\right)+\rho_{l} \mathbf{m}(\mathbf{m} \cdot \mathbf{j})+ \\
+n\left[\rho_{H} \mathbf{m}_{\|}(\mathbf{m} \cdot \mathbf{j})-\rho_{H t} \mathbf{m}\left(\mathbf{m}_{\|} \cdot \mathbf{j}\right)\right] \\
\rho_{t} \equiv \beta \eta_{l} ; \rho_{l} \equiv \beta \eta_{t} ; \rho_{H l} \equiv \beta \alpha_{H l} ; \rho_{H t} \equiv \beta \alpha_{H t} \\
\beta \equiv B \Phi_{0} / \Delta c^{2}
\end{gathered}
$$

Note that four phenomenological resistivities may be measured in two special, «basic» measurements, namely, $\rho_{l}$ and $\rho_{H l}-$ in $\mathbf{j} \perp$ TB (twin boundary) geometry, and $\rho_{t}, \rho_{H t}-$ in $\mathbf{j} \| \mathrm{TB}$ geometry. Physically, relation (6) shows how to express $\mathbf{E}$ for arbitrary angle between $\mathbf{j}$ and $\mathbf{m}$ in terms of four basic resistivities. It was postulated earlier in Ref. 2 
on the basis of the symmetry considerations. In our approach we are able to elucidate the origin of the above-mentioned resistivities [see Eq. (7)] in terms of the drag and pinning viscosities, i.e., at a more detailed level. Equation (6) admits another representation of $\mathbf{E}$ in terms of two mutually perpendicular unit vectors $\mathbf{e}_{\|} \equiv \mathbf{j} / j$ and $\mathbf{e}_{\perp} \equiv(\mathbf{z} \times \mathbf{j}) / j$; then $\mathbf{E} \equiv E_{\|} \mathbf{e}_{\|}+E_{\perp} \mathbf{e}_{\perp}$, where

$$
E_{\|}=\left(\rho_{\|}^{+}+n \rho_{\|}^{-}\right) j ; \quad E_{\perp}=\left(\rho_{\perp}^{+}+n \rho_{\perp}^{-}\right) j .
$$

Here $E_{\|}$and $E_{\perp}$ are the longitudinal (dissipative) and transverse (Hall, nondissipative) components of the electric field (in relation to the transport current density), respectively, and

$$
\left\{\begin{array}{l}
\rho_{\|}^{+} \equiv x^{2} \rho_{l}+y^{2} \rho_{t}, \\
\rho_{\perp}^{+} \equiv x y\left(\rho_{t}-\rho_{l}\right),
\end{array}, \quad\left\{\begin{array}{l}
\rho_{\perp}^{-} \equiv x^{2} \rho_{H l}+y^{2} \rho_{H t} \\
\rho_{\|}^{-} \equiv x y\left(\rho_{H l}-\rho_{H t}\right),
\end{array}\right.\right.
$$

where $x \equiv \mathbf{m} \cdot \mathbf{e}_{\|}$and $y \equiv \mathbf{m}_{\|} \cdot \mathbf{e}_{\|}$. The angle-dependent resistivities $\rho_{\|}^{ \pm}$and $\rho_{\perp}^{ \pm}$(in contrast to the «intrinsic» parameters $\rho_{l}, \rho_{t}, \rho_{H l}$, and $\rho_{H t}$ ) are measurable values for a given sample and they do not depend on the value of $n$. The sign $(+)$ here means that this value is even with respect to the reversal of the magnetic field direction, where the sign $(-)$ means that the value $n \rho_{\perp, \|}^{-}$is odd.

In the isotropic limit $\varepsilon=0$; then $\eta_{t}=\eta_{l}=\tilde{\eta} \equiv \eta+\gamma_{i}(v)$ and $\alpha_{H l}=\alpha_{H t}=\alpha$. In the limit $\alpha^{2}<<\tilde{\eta}^{2}$ we obtain directly from Eqs. (3) the results of Ref. 1 [including the nonlinear scaling relation $\rho_{x y}=\rho_{x x}^{2}\left(\alpha c^{2} / B \Phi_{0}\right)$; Eq. (7) in Ref. 1].

In contrast with the isotropic limit, where only $\rho_{\|}^{+}$and $\rho_{\perp}^{-}$are not equal to zero, in general $(\varepsilon \neq 0)$, as we can see from (9), two new contributions to the resistivity appear: transverse even $\rho_{\perp}^{+}$and longitudinal odd $n \rho_{\|}^{-}$. Note that the angular dependence of these two contributions is the same and has the maximum value at $\theta=\pi / 4$ (here $\theta$ is the acute angle between $\mathbf{m}$ and $\mathbf{j}$ ). But unlike this similarity, these contributions (as can be seen from their derivation) have a completely different origin.

The even transverse resistivity $\rho_{\perp}^{+}$stems from the evidently different pinning force for the motion of the vortices along and across the twins, and we can see in the experiment [5] the different critical currents for these two directions. Usually, in some temperature interval [5] $\rho_{t}<<\rho_{l}$, and such inequality promotes the so-called guided vortex motion [4], where the vortices prefer to move mostly along the twins than to slip across them. If we define the quantitative measure of guiding as $\cot \varphi \equiv\left|E_{\perp}^{+} / E \|\right|=\left|\rho_{\perp}^{+} / \rho_{\|}^{+}\right|$, then $\cot \varphi=$ $=(1-u) \tan \theta /\left(u+\tan ^{2} \theta\right)$ where $u \equiv \rho_{t} / \rho_{l}$ and $0<u<1$. If $u \rightarrow 0$, then $\varphi \rightarrow \theta$ and we have a full guiding. From the above formula follow several conclusions with a simple physical interpretation:

a) at $0<u<1$ always $\tan \varphi>\tan \theta$ and, in addition, $\tan \varphi$ increases when $\tan \theta$ is increasing with a fixed value of $u$,

b) if we assume $u=$ const and change $\tan \theta$, then $\tan \varphi$ as a function of $\tan \theta$ is convex downwards and corresponding minimal value of $(\tan \theta)_{\min }=2 \sqrt{u}(1-u)$ is attained at $\tan \theta=\sqrt{u}$,

c) if $\tan \theta>1$, then always $\cot \varphi<1$. But when $\tan \theta<<1$, then depending on the relation between $u$ and $\tan \theta$ we have several opportunities:

$$
\begin{aligned}
& u>\tan \theta \\
& \tan ^{2} \theta<<u<\tan \theta, \\
& u<<\tan ^{2} \theta
\end{aligned}
$$

We see that in the cases (10b) and (10c) always $E_{\perp}^{+}>>E_{\|}^{+}$. The last situation is real, because in the experiment in Ref. $5 u$ may be well below unity (for example, $u<10^{-6}$ at $T \approx 87 \mathrm{~K}$ for YBCO; see Fig. 1, curves 1 and 5 in Ref. 5).

The appearance of the odd longitudinal contribution $\rho_{\|}^{-}$, as we see from Eqs. (2), (4), (7), and (9), follows from the assumed influence of twins on the «bare» Hall drag coefficient $\alpha$. It must be stressed that such influence should be different for the vortex motion along and across the twins $\left(\gamma_{l} \neq \gamma_{t}\right)$. Physically, it may follow from a different behavior of the Magnus force, whose microscopic origin may be highly complex (see, for example, Ref. 6). It is evident, at least, that the vortices move along the twin at the constant value of the order parameter, whereas the order parameter value is not homogeneous for the vortices that move across the twins.

3. In what follows, however, we consider another mechanism for the $\rho_{\|}^{-}$appearance, which implies that the Ettingshausen effect is possible. For this reason, we will initially discuss the case in which, the transport current is zero, but there is a thermal force

$$
\mathbf{f}_{T}=s_{\|} \nabla T+s_{\perp} \nabla T \times \mathbf{n} .
$$

Here $\nabla T$ is the temperature gradient vector in the plane of the sample, and $s_{\|}$and $s_{\perp}$ are the pheno- 
menological constants $\left(s_{\|} \equiv S_{\varphi}\right.$ is the transport entropy per vortex unit length, $s_{\perp} \equiv \Phi_{0} Q_{n} / \rho_{n}$, where $Q_{n}$ is the normal state thermoelectric power, and $\rho_{n}$ is the normal state resistivity; see, for example, Ref. 7). Substitution of the thermal force components $f_{t}^{T}$ and $f_{l}^{T}$ (instead of $f_{t}$ and $f_{l}$ ) into Eqs. (3)

$$
\begin{aligned}
& f_{t}^{T} \equiv s_{\|} x_{T}+n s_{\perp} y_{T}, \\
& f_{l}^{T} \equiv s_{\|} y_{T}-n s_{\perp} x_{T},
\end{aligned}
$$

$$
\left\{\begin{array}{c}
s_{\|}^{t} \equiv(B / c \Delta)\left(\eta_{l} s_{\|}+\alpha_{H l} s_{\perp}\right), \\
s_{\perp}^{t} \equiv(B / c \Delta)\left(\eta_{l} s_{\perp}-\alpha_{H l} s_{\|}\right),
\end{array}\right.
$$

Finally, we have the following equation for the electric field $\mathbf{E}$ produced by $\nabla T$ [compare with Eq. (6)]:

$$
\begin{aligned}
& \mathbf{E}_{T}=s_{\perp}^{t} \mathbf{m}_{\|}\left(\mathbf{m}_{\|} \cdot \nabla T\right)+s_{\perp}^{l} \mathbf{m}(\mathbf{m} \cdot \nabla T)+ \\
& \left.+n\left[s_{\|}^{t} \mathbf{m}_{\|}(\mathbf{m} \cdot \nabla T)-s_{\|}^{l} \mathbf{m}^{\left(\mathbf{m}_{\|}\right.} \cdot \nabla T\right)\right] .
\end{aligned}
$$

Comparing (15) and (6), we see that if we change $s_{\perp} \rightarrow\left(\Phi_{0} / c\right), s_{\|} \rightarrow 0$, and $\nabla T \rightarrow \mathbf{j}$, then Eq. (15) transforms into Eq. (6). All physical analysis of Eq. (6) can therefore be repeated for Eq. (15) with only one essential distinction: for thermomagnetic effects all four parameters given by (14) are the values of the same order of magnitude [7], because $s_{\|}$and $s_{\perp}$ in Eq. (11) are approximately of the same order of magnitude for HTSC. Note, however, that in Eq. (6) $\rho_{H l} \ll<\rho_{l}$ and $\rho_{H t} \ll<\rho_{t}$. From Eqs. (14) and (15) it follows that the Hall contributions $\left(\sim \alpha_{H t}\right.$ and $\left.\alpha_{H l}\right)$ always are small in comparison with other terms which are proportional to $\eta_{t}$ and $\eta_{l}$. Because of this circumstance, all new (i.e., those stemming from anisotropy) thermomagnetic effects, giving $E_{\|}^{-}(\nabla T)$ and $E_{\perp}^{+}(\nabla T)$, might be of the same order of magnitude [unlike galvanomagnetic effects, where usually $\left.E_{\|}^{-}(j) \ll E_{\perp}^{+}(j)\right]$. Until now, several interesting experimental investigations of the thermomagnetic effects in YBCO single crystals with unidirected twins have been carried out [8]. But below we deal only with «secondary» thermomagnetic effects produced by $\nabla T$, which, in turn, is generated by the transport current density $\mathbf{j}$ due to the Ettingshausen effect (the latter in almost adiabatic conditions was measured in YBCO by Battlogg et al. [9]). In the case of anisotropic pinning such «secondary» thermomagnetic effects produce additional odd longitudinal («Seebeck») and even transverse ( $\ll$ Nernst») contributions to the where $f_{t}^{T} \equiv \mathbf{m} \cdot \mathbf{f}_{T}, f_{l}^{T} \equiv \mathbf{m}_{\|} \cdot \mathbf{f}_{T}$ and $x_{T} \equiv \mathbf{m} \cdot \nabla T$, $y_{T} \equiv \mathbf{m}_{\|} \cdot \nabla T$ leads to the solutions of Eqs. (3) in the form (5)

$$
\begin{aligned}
& v_{t}^{T}=(c / B)\left(s_{\|}^{t} x_{T}+n s_{\perp}^{t} y_{T}\right), \\
& v_{l}^{T}=(c / B)\left(s_{\|}^{l} y_{T}-n s_{\perp}^{l} x_{T}\right),
\end{aligned}
$$

where

$$
\left\{\begin{array}{c}
s_{\|}^{l} \equiv(B / c \Delta)\left(\eta_{t^{s}} s_{\|}+\alpha_{H t} s_{\perp}\right), \\
s_{\perp}^{l} \equiv(B / c \Delta)\left(\eta_{t^{\prime}} s_{\perp}-\alpha_{H t^{s} \|}\right) .
\end{array}\right.
$$

measured voltages. If the real experiment is carried out in nonisothermal conditions, these additional contributions may mask the «intrinsic» odd longitudinal contribution which is attributable to the possible influence of the twins on the Hall drag coefficient. Moreover, it is conceivable that in the case of bad heat removal conditions the intrinsic $E_{\|}^{-}=0$ (i. e., $\alpha_{H l}=\alpha_{H t}=\alpha$ ), but we measure only $\delta E_{\|}^{-}$produced by the Ettingshausen effect. Analysis of the experimental observations of $E_{\|}^{-}$(in contrast to $E_{\perp}^{+}$) therefore requires more accurate estimates of the heat removal conditions, especially for the case of large transport current densities. Theoretical estimate of the additional odd longitudinal contribution $\delta E_{\|}^{-}$in the adiabatically isolated sample can be derived as follows. First, we calculate $\nabla T_{L}$, which arises due to the heat current flow density $\mathbf{Q}_{\varphi}$ carried by the vortices which move with the velocity $\mathbf{v}_{L}$ in the sample with the anisotropic pinning in the presence of the transport current density $\mathbf{j}$

$$
\mathbf{Q}_{\varphi}=U_{\varphi} \mathbf{v}_{L}=T S_{\varphi} \mathbf{v}_{L}=-\kappa \nabla T_{L} .
$$

Here $U_{\varphi}=T S_{\varphi}$ is the thermal energy of the vortices, and $\kappa$ is the thermal conductivity of the sample. From (16) we have

$$
\nabla T_{L}=-\mu \mathbf{v}_{L}=-(\mu c / B)\left(\mathbf{n} \times \mathbf{E}_{L}\right) .
$$

Here $\mu \equiv T S_{\varphi} / \kappa$ and $\mathbf{E}_{L}$ is given by Eq. (6). Substitution of (17) into the equation for $\mathbf{E}_{T}$ (15) after some calculations gives us the desired additional odd longitudinal contribution $\delta E_{\|}^{-}$:

$$
\delta E_{\|}^{-}=(\mu c / B j)(x y)\left[\left(s_{\perp}^{l} \rho_{t}-s_{\perp}^{t} \rho_{l}\right)+\left(s_{\|}^{t} \rho_{H l}-s_{\|}^{l} \rho_{H t}\right)\right] .
$$

Neglecting a small Hall contribution in the second part of the brackets and comparing (18) with the 
equation for $E_{\|}^{-}(8)$, we see that both contributions have the same angular dependence and may have the same order values.

For further estimates we can use (18), in which we replace $\kappa$ by $\kappa_{\text {eff }}$ in order to take into account the actual heat-transfer conditions ( $\kappa_{\text {eff }}$ is always greater than $\kappa$ ). If the heat removal from the sample is effective (in term of $\kappa_{\text {eff }}$ it means that $\kappa_{\text {eff }} \rightarrow \infty$ ), then $\delta E_{\|}^{-} \rightarrow 0$ and we can ignore it. In the opposite case $\left(\kappa_{\text {eff }} \rightarrow \kappa\right)$ we would have $\delta E_{\|}^{-}>>E_{\|}^{-}$.

In conclusion, we stress that Eqs. (3) allow us to consider nonlinear galvanothermomagnetic effects. This will be the subject of the next publication.

It is my pleasure to thank A. A. Prodan for help in preparing this paper for publication.

1. V. M. Vinokur, V. B. Geshkenbein, M. V. Feigelman, and G. Blatter, Phys. Rev. Lett. 71, 1242 (1993).
2. E. B. Sonin and A. L. Kholkin, Fiz. Tverd. Tela 34, 1147 (1992). [Sov. Phys. Solid State 34, 610 (1992)]; E. B. Sonin, Phys. Rev. B48, 10487 (1993).

3. A. V. Samoilov, A. Legris, F. Rulier-Albenque, P. Lejay, S. Bouffard, Z. G. Ivanov, and L.-G. Johansson, Phys. Rev. Lett. 74, 2351 (1995).

4. A. K. Niessen and C. H. Weijsenfeld, J. Appl. Phys. 40 384 (1969)

5. A. V. Bondarenko, M. A. Obolenskii, R. V. Vovk, A. A. Prodan, V. A. Shklovskij, and A. G. Sivakov, in: Proc. of the 7th Intern. Workshop on Critical Currents in Superconductors, H. W. Weber (ed.) World Scientific, Singapore, (1994), p. 177.

6. E. B. Sonin, Phys. Rev. B55, 485 (1997).

7. T. W. Clinton, Wu Liu, X. Jiang, A. W. Smith, M. Rajesuari, R. L. Greene, and C. J. Lobb, Phys. Rev. B54, R9670 (1996).

8. H. Ghamlouch and M. Aubin, Physica C269, 163 (1996); H. Ghamlouch, M. Aubin, R. Gagnon, and L. Taillefer, Physica C275, 141 (1997).

9. T. T. M. Palstra, B. Batlogg, L. F. Schneemeyer, and J. V. Waszczak, Phys. Rev. Lett. 64, 3090 (1990). 